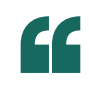

The poor understanding of its molecular mechanisms is probably the reason why hydrotropy is often overlooked when debating the future of green chemistry
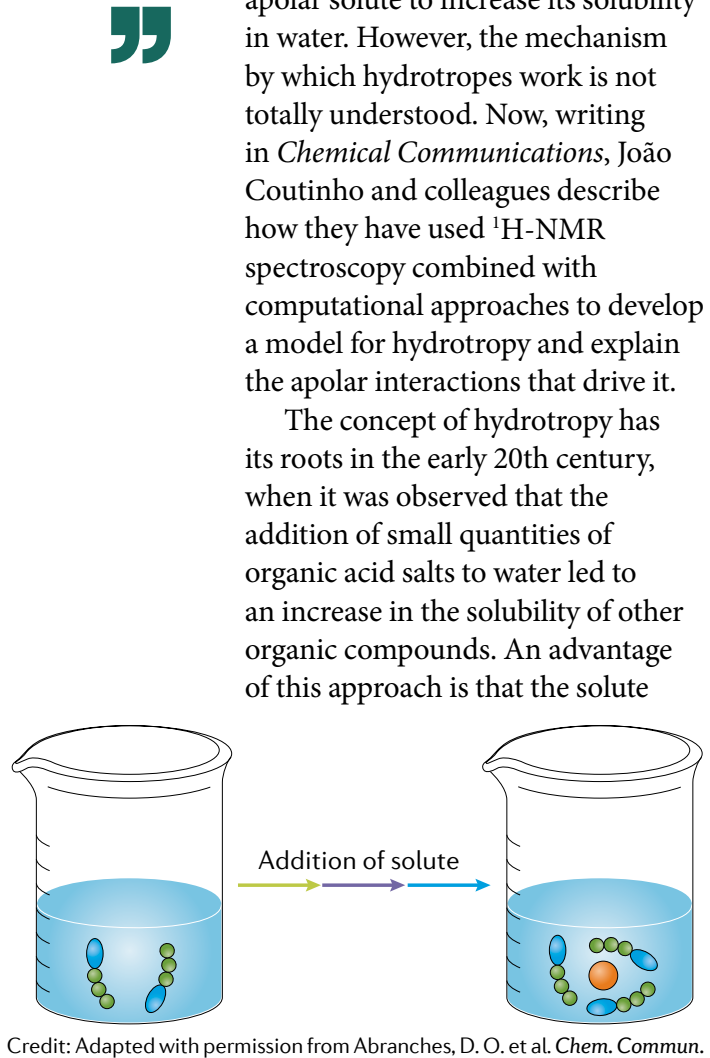
https://doi.org/10.1039/D0CC03217D (2020), RSC

If water were able to dissolve both polar and apolar solutes, then this one solvent alone might suffice for all applications. Although such a goal may seem utopian, chemistry offers some clever ways to assist in solubilizing apolar molecules in water. Surfactants, for example, have a polar head and an apolar tail and, as a result, assemble into micelles that can accommodate the apolar solute within the core while becoming homogeneously dispersed in water. Hydrotropes are similar to surfactants in that they have both polar and apolar components, but they do not aggregate into micelles - they interact directly with the apolar solute to increase its solubility in water. However, the mechanism by which hydrotropes work is not totally understood. Now, writing in Chemical Communications, João Coutinho and colleagues describe how they have used ${ }^{1} \mathrm{H}-\mathrm{NMR}$ spectroscopy combined with computational approaches to develop a model for hydrotropy and explain the apolar interactions that drive it.

The concept of hydrotropy has its roots in the early 20 th century, when it was observed that the addition of small quantities of organic acid salts to water led to an increase in the solubility of other organic compounds. An advantage of this approach is that the solute

\title{
HYDROTROPY
}

\section{Solving a solubility problem}

can be recovered by lowering the concentration of the hydrotrope, thus avoiding the need for complex separation processes. Since then, the mechanism of hydrotropy has been rationalized in many ways. For example, it has been suggested that it may be driven by the self-aggregation of the hydrotropes, by disruption of the water structure or by pure stoichiometric arguments. "The poor understanding of its molecular mechanisms is probably the reason why hydrotropy is often overlooked when debating the future of green chemistry," says Coutinho.

The team led by Coutinho wanted to provide a rational mechanism for hydrotropy that agrees well with the statistical thermodynamic picture according to which hydrophobic interactions result in a stabilizing arrangement of the hydrotropes around the apolar solute molecules. "Our work builds on the efforts of Professor Seishi Shimizu and co-workers, who, for the past few years, have been tackling the foundations of hydrotropy using statistical thermodynamics," explains Coutinho. "Our collaboration with the research group led by Elísabet Pires, who have a long history in designing and synthetizing tailor-made glycerol ethers, provided access to a plethora of these compounds with specific alkyl side chain lengths, allowing us to perform a consistent study on hydrotropy, probing the effect of the apolarity by gradually increasing the apolar nature of the hydrotrope." Achieving this degree of understanding of the hydrotropy mechanism might eventually enable a rational choice of solute-hydrotrope combinations for specific applications.
Coutinho and co-workers performed ${ }^{1} \mathrm{H}-\mathrm{NMR}$ spectroscopy measurements to monitor the interactions between monoalkylglycerol ethers of different polarity and two water-insoluble organic acids - gallic acid and syringic acid. The chemical shift of the apolar protons in the glycerol ether hydrotropes was found to decrease with increasing solute concentrations. This indicates that the apolar moiety of the hydrotope is shielded from water by the solute. This rules out the possibility of self-aggregation of the hydrotrope, which would have not induced any significant change in the chemical shift of these protons.

The experimental results were then rationalized by quantifying the molecular surface corresponding to a polarization charge density, $\sigma$. An analysis of the $\sigma$-profiles revealed that solute solubility increases with the increase of the apolar surface area of the hydrotrope and it reaches a maximum when the apolar surface area of the hydrotrope and solute match. A further increase of the apolar surface area of the hydrotrope leads, instead, to its self-aggregation.

"Perhaps the most significant finding of our work is the mental picture that we can now build of hydrotropy," says Coutinho. "In our opinion, there are a number of remaining fundamental questions. Despite its age, the concept of hydrotropy remains poorly understood and is much underexplored," he concludes.

Gabriella Graziano

ORIGINAL ARTICLE Abranches, D. O. et al. Unveiling the mechanism of hydrotropy: evidence for water-mediated aggregation of hydrotropes around the solute. Chem. Commun. https://doi.org/ 10.1039/D0CC03217D (2020) RELATED ARTICLE Booth, J. J., Abbott, S. \& Shimizu, S. Mechanism of hydrophobic drug solubilization by small molecule hydrotropes. J. Phys. Chem. B 116, 14915-14921 (2012) 